
internationales

vol. 24 - $n^{\circ} 3 \mid 2008$

Numéro ouvert

\title{
Séparées et réunies : familles migrantes et liens
} transnationaux

Separated and Reunited: Migrant Families and Transnational Bonds

Separadas y reunidas: familias migrantes y lazos transnacionales

\section{Maurizio Ambrosini}

\section{CpenEdition}

Édition électronique

URL : https://journals.openedition.org/remi/4829

DOI : $10.4000 /$ remi. 4829

ISSN : $1777-5418$

Éditeur

Université de Poitiers

Édition imprimée

Date de publication : 1 décembre 2008

Pagination : 79-106

ISBN : 978-2-911627-50-7

ISSN : 0765-0752

\section{Référence électronique}

Maurizio Ambrosini, «Séparées et réunies : familles migrantes et liens transnationaux », Revue

européenne des migrations internationales [En ligne], vol. 24 - n³ | 2008, mis en ligne le 01 décembre

2011, consulté le 14 avril 2022. URL : http://journals.openedition.org/remi/4829 ; DOI : https://doi.org/ $10.4000 /$ remi. 4829 


\title{
Séparées et réunies : familles migrantes et liens transnationaux
}

\author{
Maurizio AMBROSINI*
}

Une des principales nouveautés dans les recherches sur les migrations internationales est la prise en compte d'une perspective transnationale. Elle considère que les migrants, acteurs sociaux dans leur espace d'accueil et d'origine, construisent et alimentent des liens entre leurs deux mondes, s'impliquant économiquement, politiquement, et culturellement dans des initiatives et des activités qui connectent les pôles de leur déplacement (Basch, Glick Schiller et Szanton Blanc, 1994 ; Portes, 1999, 2003 ; Levitt et Jaworsky, 2007 ; Ambrosini, 2008).

Le transnationalisme comme choix intentionnel et comme stratégie poursuivie par les migrants ne doit pas cacher un autre aspect des relations transnationales, moins voulu que subi, lié au nombre croissant de personnes et de familles qui en dépit des frontières et des distances qui les séparent, s'efforcent de maintenir des liens affectifs et d'assumer leurs responsabilités parentales dans le cadre de «familles transnationales ». Ce transnationalisme actif et dynamique conteste les prétentions d'assimilation des États-nations en construisant des communautés unies, solidaires malgré les distances physiques. Bien qu'il se teinte des couleurs mélancoliques de l'absence et de la nostalgie, ce sont les tons vibrants de la détermination qui l'emportent et qui permettent de maintenir vivantes les relations avec les personnes qui sont au loin. C'est justement le lien affectif et le désir d'assurer aux siens une vie meilleure qui conduit à cette forme extrême de dévouement et qui se traduit par un éloignement physique. Il est vrai qu'il ne s'agit pas d'un « transnationalisme forcé » car, dans ce cas, les personnes choisissent, investissent, élaborent et poursuivent de façon tenace des objectifs d'amélioration et des espoirs de promotion. Il ressemble plus à une adaptation à un ensemble de nécessité et de limites qu'à une perspective de

* Professeur associé à l'Université de Gènes, Dipartimento di Scienze Antropologiche, sezione di Sociologia, via Lomellini 3, 1-16124 Genova, maurizio.ambrosini@unimi.it 
liberté d'action qui dépasse les frontières. Néanmoins, à travers ce vécu de privation et de souffrance, en persévérant dans la prise en charge à distance, s'ourdit la texture des liens qui dépassent les frontières, en alimentant la bifocalité (Vertovec, 2004 a) et la densité relationnelle de l'expérience migratoire.

Il faut préciser que, comme pour d'autres phénomènes aujourd'hui définis comme transnationaux, la situation des familles séparées par l'émigration d'un ou plusieurs de leurs membres n'est pas nouvelle dans l'histoire des migrations. Le cas des « veuves blanches » ${ }^{1}$ des émigrants reste ancré dans la mémoire collective de nombreuses sociétés d'origine.

Tant que les émigrants étaient les membres masculins de la famille - époux, pères, fils - les études n'avaient pas identifié une forme familiale émergente comme produit des migrations, et n'avaient pas non plus thématisé une souffrance particulière des sujets impliqués, sauf dans le cas des «veuves blanches ». Les attentes réciproques concernant la courte durée de l'émigration et des rôles sociaux codifiés, selon lesquels les soins apportés à la famille étaient associés à la figure maternelle, alors que sa subsistance concernait les pères, conduisaient à considérer comme socialement acceptable l'éloignement des pères et des maris : le migrant pouvait se considérer et être vu comme un bon père et un bon mari en partant pour assurer le bien-être des siens.

L'image des familles transnationales a été bouleversée avec la fréquence croissante des départs des femmes qui confient leurs enfants dans un premier temps à leurs propres mères (Hondagneu-Sotelo et Avila, 1997 ; Dreby, 2006), puis à leurs sœurs, leurs filles majeures, plus rarement leur mari, parfois à des femmes rémunérées. Se construit alors une sorte de chaîne où l'émigrante confie à d'autres femmes la charge des soins familiaux. Ainsi pour Mahler les femmes sont les figures clés dans la gestion et le soutien des familles transnationales (1998). Bien que les mères transnationales soient activement impliquées dans l'exercice de la maternité s'efforçant d'apporter à leurs enfants affection et soutien, la perte de la proximité physique est ressentie, par elles-mêmes et par tous les membres de leur famille comme la violation d'un modèle profondément légitime, celui de la mère qui prend en charge ses propres enfants (Hondagneu-Sotelo et Avila, 1997).

La perception diffuse d'une anomalie dans le fonctionnement de ces familles est liée au sentiment de vide qu'entraîne le départ de la mère biologique, celle dont on attend, dans presque toutes les cultures, qu'elle assure l'essentiel des soins de ses enfants. C'est autour de cette absence que se structure le discours relatif aux familles transnationales, à leur souffrance et à leurs méthodes de compensation. Ceux-là mêmes qui comme Parreñas (2001) définissent la famille transnationale comme « une structure familiale postindustrielle avec des valeurs préindustrielles », ne peuvent nier le bouleversement des pratiques de prise en charge profondément enracinées.

1 On appelait veuves blanches les femmes dont les maris émigraient à l'étranger et qui, après un certain temps, cessaient de donner des nouvelles. 
Cette analyse s'inscrit dans une thématique plus large, celle de l'entrelacement de l'institution familiale avec les processus migratoires, dans lequel les familles transnationales peuvent constituer de façon alternative une forme dynamique et variable de la famille, ou encore une phase de la vie familiale. Dans les deux cas, il convient de s'interroger sur les vécus quotidiens des hommes et des femmes migrantes : espoir, douleur, détachement, retrouvailles et enfin construction de l'avenir dans des conditions difficiles. Sur un plan plus théorique, la notion de « familles transnationales » contribue à mettre l'accent sur la famille comme acteur intermédiaire entre l'individu migrant et les grands processus structurels qui mettent en mouvement les migrations, en la situant dans un contexte de relations, d'une ampleur croissante, avec des systèmes parentaux et des réseaux migratoires plus étendus (Ambrosini, 2005).

Dans cet article, j'aborderai dans un premier temps la demande de maind'œuvre féminine en Italie et en Europe méridionale, pour répondre aux exigences croissantes des activités de soin et de prise en charge des membres des familles, demande qui conduit à une "stratification internationale des opportunités de soins et d'assistance ». Je m'intéresserai ensuite aux modalités par lesquelles les mères séparées de leurs enfants s'efforcent de continuer à exercer leurs devoirs maternels, malgré les frontières et les distances. Je mettrai en évidence la diversité de leurs comportements en présentant une typologie des familles transnationales. Mais le phénomène n'est pas rigide et bloqué, il présente une composante évolutive, qui transforme une partie des familles transnationales en familles réunies, dans lesquelles surgissent d'ailleurs de nouveaux problèmes. Pour conclure, je discuterai les implications théoriques, politiques et sociales de la question abordée.

\section{LES RACINES DU PHÉNOMÈNE : LES FEMMES IMMIGRÉES, RESSOURCE DU WELFARE INFORMEL}

Aborder le thème des familles transnationales demande d'abord une réflexion sur la relation entre immigration (le plus souvent, mais pas seulement féminine) et assistance à la personne, dans le domaine de ce qui peut être défini comme celui du welfare informel, caché, invisible. Particulièrement fréquent en Europe méridionale, il est présent sous d'autres formes dans d'autres pays d'Europe ${ }^{2}$, aux États-Unis et dans les villes des pays émergents. Il prolonge le rôle traditionnel de prise en charge attribué aux familles (et aux femmes qui la composent) qui, ne pouvant plus faire face à la pression de la demande avec leurs seules forces, tendent (ou sont obligées) à réaffirmer la fonction propre de pivot du bien-être familial en ayant recours à des collaborateurs, bonnes d'enfants ou assistantes à domicile pour les personnes âgées. Il ne s'agit pas d'un phénomène nouveau dans l'histoire des migrations. Comme le rappelle Amalia Signorelli, « il s'agit d'un mouvement constant de filles pauvres de la campagne vers les villes de l'Occident, mouvement qui a duré pendant des siècles et a parfois atteint des dimensions impressionnantes comme celles enregistrées par les Irlandaises qui

2 Cf. Anderson, 2000. 
durant la grande famine partaient s'engager comme servante en Angleterre et aux États-Unis. Tout cela a été refoulé, jugé insignifiant, oublié » (2006 : 26). Tout au plus, peut-on parler aujourd'hui d'un retour de rapports sociaux et de travail préindustriels dans une société postindustrielle, retour ne concernant pas seulement les familles aisées mais connaissant une large diffusion sociale.

La forme silencieusement acquise par le système sud-européen des soins à domicile s'est révélée cohérent avec le modèle familial du welfare caractérisant l'Italie et des autres pays méditerranéens d'Europe, système mis en relief de façon particulière par Esping-Andersen (2000). Dans le cadre familial, diverses tâches sont implicitement déléguées aux femmes alors qu'elles sont prises ailleurs en charge par les institutions publiques. Actuellement cette organisation est de plus en plus bousculée depuis l'entrée massive des épouses sur le marché du travail, alors que le nombre des personnes âgées ayant besoin d'assistance augmente et alors que la redistribution des tâches domestiques à l'intérieur de la famille n'a pas progressé ${ }^{3}$.

L'embauche de femmes immigrées (et parfois aussi d'hommes) comme collaborateurs familiaux et assistantes à domicile peut être vu comme une ressource pour alléger les charges domestiques des familles (et notamment des épouses). La disponibilité d'une offre de travail abondante, très flexible et peu coûteuse comme celle des femmes immigrées en situation irrégulière, a joué un rôle décisif dans la structuration sous forme privée de la demande d'assistance et de prise en charge à domicile.

C'est ainsi que prend forme un welfare « léger », familial et informel ${ }^{4}$, peu professionnalisé et vécu comme plus «amical », sans bureaucratie, flexible, et naturellement plus gérable par les utilisateurs-employeurs. Les familles renoncent à profiter de services institutionnels (qui du reste ne pourraient pas répondre à leurs besoins) ainsi qu'à une assistance professionnellement qualifiée et rationnellement organisée pour gérer plus librement l'assistance aux personnes âgées dans un cadre domestique, en évitant de bouleverser les habitudes et les rythmes de vie du conjoint.

Un autre aspect est aussi à considérer sur un plan plus théorique, lequel fait référence aux catégories de réciprocité, de redistribution et de marché (Polanyi, 1974 ; Cella, 1997). L'assistance aux personnes dépendantes, réglée le plus souvent en Europe du Sud selon le code de la réciprocité, est basée sur la solidarité familiale et sur les tâches liées aux soins traditionnellement attribuées aux femmes ; les mesures de redistribution d'initiative publique (assistance à domicile, hospitalisation dans des

3 Les données sur les structures et les services pour l'assistance aux personnes âgées non autonomes ne sont pas homogènes et sont plutôt discordantes. Cependant, on peut définir clairement trois aspects : l'Italie, avec $20 \%$ de personnes âgées, est un des pays du monde dans lesquels le vieillissement est le plus prononcé ; 2) le nombre de lits dans les maisons de retraite est un des plus faibles parmi les pays développés ; 3) l'aide à domicile gérée par les organismes sociaux (qui ne résout que difficilement et partiellement le problème de la perte d'autonomie) est très peu développée.

4 Cf. Tognetti Bordogna, 2004. 
structures protégées) sont considérées comme résiduelles, destinées aux personnes âgées pauvres et seules, privées de réseau familial capable de les protéger. De façon plus générale, l'action publique a préféré se fonder sur des transferts de revenus destinés à reconnaître et renforcer les capacités d'assistance des familles, c'est-à-dire les structures de la réciprocité. Les familles, pourtant, grâce aussi aux ressources économiques distribuées par le système public, ont entrepris une restructuration silencieuse de la réglementation de l'assistance aux personne âgées, en instituant un marché privé des soins à domicile largement informel, avec l'embauche de personnes (femmes ou hommes étrangers) pour remplacer ou intégrer des ressources intrafamiliales (et féminines) de réciprocité insuffisante. Mais il ne s'agit pas d'un simple remplacement de la réciprocité par le marché : les assistants à domicile rétribués ont tendance à être incorporés et encadrés, à leur tour, à travers les normes d'une sorte de réciprocité familiale élargie, qui entraîne une profonde ambivalence.

E. Colombo observe que : «Être étranger est l'une des caractéristiques qui permettent de réduire les tensions liées à l'asymétrie du pouvoir de qui est dans la condition de faire faire à d'autres des tâches que lui-même n'est pas disposé à faire personnellement. L'asymétrie de l'étranger justifie l'asymétrie des relations de pouvoir » (2007 : 117). En même temps se produisent des dynamiques relationnelles qui incluent, d'une certaine façon, l'aide à domicile immigrée dans la famille. Son intégration est une attente implicite et non un aspect défini au moment où se négocient les conditions du contrat de travail. On attend une implication affective et une disponibilité à l'écoute et au soutien psychologique des assistés. De plus, l'encadrement cognitif de l'aide à domicile fonctionne mieux sur le versant des devoirs que sur celui des droits : il peut devenir une modalité tactique pour donner à la personne la responsabilité des tâches de compagnie, de surveillance, d'assistance, qui dépassent les horaires et les fonctions contractuelles.

Par ailleurs la personne embauchée peut elle aussi rechercher et apprécier cette intégration dans la famille, ou du moins l'accepter. Séparée des siens et de son espace de vie, elle peut trouver consolant qu'une famille lui offre non seulement un travail, mais aussi un lieu accueillant. Une des formes les plus courantes d'adaptation à la situation se traduit, lorsqu'elle prend en charge des enfants, de s'y attacher et de compenser ainsi partiellement l'impossibilité de voir grandir ses propres enfants. On constate également des reproches et des formes de compétitions à l'égard des mères pourvoyeuses d'emploi, jugées trop occupées et peu disponibles pour leurs enfants (Hondagneu-Sotelo et Avila, 1997). De plus, dans certaines situations, les familles qui ne sont pas satisfaites des prestations de l'assistante à domicile ou de la nourrice, évitent cependant de les licencier s'ils perçoivent que la personne âgée ou l'enfant y sont attachées.

L’intégration dans la famille est étroitement liée à la cohabitation. Elle n'est pas absente d'avantages pour les acteurs concernés, mais elle représente un terrain susceptible d'interprétation et de confusion dans ce qui reste un contrat de travail. C'est précisément la fin du contrat, notamment quand c'est l'employée qui le décide, qui révèle l'ambiguïté de la situation : elle rompt l'intégration dans la famille et ramène les rapports à un échange professionnel. L'employée, même attachée aux personnes qu'elle 
assiste fera remarquer qu'elle a sa propre famille et qu'elle doit y penser, en acceptant peut-être un travail mieux rémunéré ou en cherchant un logement personnel, posant ainsi les bases d'un regroupement familial.

\section{MÈRES À DISTANCE : LA STRATIFICATION INTERNATIONALE DES OPPORTUNITÉS D'ASSISTANCE}

Le rôle que les femmes immigrées, souvent épouses ou mères, assument dans notre système du welfare informel, met sous tension leur organisation familiale, en obligeant ses membres à se réadapter et à compenser de diverses façons. C'est ainsi que se crée une stratification internationale des opportunités d'assistance ${ }^{5}$. Au sommet on y trouve les familles aisées des pays développés, assistées par des employés de maison, et à la base les familles transnationales des pays pauvres, qui doivent combler avec des solutions tampons le départ des mères qui vont à l'étranger pour prendre soin de personnes âgées et d'enfants ; les familles sont à leur tour différenciées et socialement stratifiées, sur la base des ressources dont elles disposent pour faire face au care drain $^{6}$. Les normes des pays d'accueil renforcent cette asymétrie : il est relativement aisé de faire venir une femme étrangère, de façon régulière ou non, pour lui confier des tâches liées à l'assistance dans une famille, en Italie et ailleurs. Par contre il est bien plus difficile pour ces femmes de recomposer leur propre famille dans le pays d'accueil, étant données les législations liées au séjour, au revenu et aux conditions de logement exigées pour tout regroupement familial. De plus, elles seront contraintes à une reconversion professionnelle, ne pouvant pas faire venir en même temps que leurs enfants une parente pour l'assister dans ses propres tâches domestiques.

L'intérêt des chercheurs pour l'implication des contextes familiaux dans les migrations féminines internationales ne s'est développé que récemment ${ }^{7}$. La littérature a mis en relief la « souffrance de la parentalité transnationale » (Parreñas, 2001), liée à ce que nous avons déjà défini comme care drain, un phénomène analogue au brain drain et au drainage historique tout d'abord de ressources naturelles, puis de maind'œuvre, par les pays riches dans leurs ex-colonies et plus généralement, dans les payses sous-développés. Aujourd'hui les familles des pays riches ôtent à celles plus pauvres une ressource bien plus subtile, liée aux tâches traditionnelles des femmes, faite de dévouement, de soins, d'une attention constante envers les besoins des autres ; une ressource qui appelle la tendresse, voire l'amour (Ehrenreich et Hochschild, 2002).

5 Je réélabore ici le concept de «stratification reproductive » proposé par Colen (1995).

6 Durant un voyage en Équateur au mois d'octobre 2005, j'ai pu observer que le départ des parents, et en particulier des mères d'enfants en bas âge est perçu à l'intérieur des communautés situées dans les zones intérieures du pays (la Sierra) les plus touchées par l'émigration externe, comme une situation d'urgence sociale. L'Église catholique, des plus hauts niveaux aux paroisses des villages, s'efforce d'accueillir et d'éduquer les enfants restés au pays, et tente de décourager - apparemment sans succès — les nouveaux départs vers les États-Unis et l'Europe.

7 En Italie, une des contributions qui ont anticipé le débat a été celle de Scabini et Donati (1993), mais elle a été peu suivie. 
L'émigration nie aux mères transnationales la possibilité de « tenir les rôles de genre » (to do gender, selon l'expression américaine) selon les normes définies culturellement : elle s'oppose directement à la possibilité pour elles de fournir les soins et d'être gardiennes des liens parentaux dans les familles et dans les communautés d'origine (Aranda, $2003: 624$ ), alors que pour les hommes, elle représente une modalité, certes coûteuse mais culturellement légitime, de décliner leur rôle socialement attendu, celui de pourvoyeurs de ressources dont leur famille a besoin. C'est pour cette raison, qu'une campagne politique et médiatique qui s'étend de l'Amérique Latine, aux Philippines, plus récemment en Europe Orientale (Castagnone et al., 2007), a mis l'accent sur les problèmes des enfants définis par certains comme " orphelins sociaux » : ils se plaignent de la solitude, de la dépression, des échecs scolaires, et on enregistre aussi des suicides, ainsi que des consommations excessives d'alcool et de drogues favorisées par une certaine disponibilité financière en l'absence du contrôle maternel. Il est aisé d'imputer ces conséquences aux mères migrantes, de leur conseiller de rentrer chez elles pour reprendre leur rôle traditionnel ${ }^{8}$. De plus, comme l'a démontré Bonizzoni (2007), les mères sont le plus souvent culpabilisées non seulement par la communauté, mais aussi par leurs enfants, qui vivent leur départ comme un abandon et non pas celui des pères.

En compensation, on ne demande pas aux mères un apport important en termes d'envois d'argent, et leur sollicitude maternelle n'est pas mesurée en fonction de la régularité ou du niveau de ses envois. Ce devoir est en effet attribué aux pères, qui ont même honte de téléphoner aux enfants, selon la recherche de Dreby, s'ils n'ont rien à envoyer. "Lorsque les mères n'envoient pas d'argent ou de dons à la maison elles n'ont pas nécessairement le sentiment d'avoir détérioré leur relation avec les enfants. Ce qui compte c'est l'expression de la sollicitude et non pas l'envoi de biens matériels. Pour les pères, au contraire, c'est ce qu'ils envoient à la maison qui compte. » (2006 : 55). Par conséquent, alors que les mères racontent leur séparation d'avec les enfants en termes de sacrifice et de douleur, il n'en va pas de même pour les pères : l'éloignement physique ne s'oppose pas au rôle qui leur est demandé, au contraire il confirme le sérieux avec lequel il est assumé.

La question du rapport entre les mères expatriées et les enfants restés au pays représente en tout cas un nœud crucial du phénomène des familles transnationales. Les oscillations de la littérature dans ce domaine sont bien visibles dans les contributions de Parreñas (2001 ; 2005). D'une part, cette auteure tend à déconstruire de façon critique les « conceptions conventionnelles » de la famille, qui impliquent des concepts d'intimité physique, mentale et affective et qui dans le cas philippin sont renforcées par des traditions culturelles où la solidarité familiale occupe le premier rang. Cependant, elle illustre la «pénible contradiction des sentiments » vécue par les mères expatriées

8 En réalité, comme l'a observé Eve (2007), en admettant que le problème de la séparation des enfants des mères transnationales persiste, en imputer la responsabilité à la mère migrante est simplificateur. Ainsi, comme le démontrent plusieurs recherches, la désagrégation ou la crise familiale peut être la cause et non l'effet du départ des femmes (Banfi et Boccagni, 2007). 
en s'arrêtant sur les tensions émotives de la parentalité transnationale qui englobent des sentiments d'anxiété, d'incapacité, de perte, de faute et de solitude. Si ces sentiments peuvent être déterminés culturellement, l'auteur va plus loin en affirmant que « dans les familles transnationales l'absence d'une interaction quotidienne empêche la familiarité et devient une lacune irréparable dans la définition des relations parentsenfants » (2001 : 121). Parreñas observe que les parents émigrés, au lieu de payer leurs dettes affectives envers les enfants en passant plus de temps ensemble, donnent de l'amour par l'intermédiaire d'achats de vêtements italiens ou américains et de matériel scolaire : « ils remplacent l'amour par les envois mensuels d'argent » (2001: 124). De diverses manières, les mères cherchent à justifier la séparation familiale : elles ne peuvent pas se permettre de se confronter avec les tensions affectives qui menacent les relations familiales même si pour la plupart les cadeaux et la communication à distance ne peuvent pas remplacer l'intimité et les interactions.

À la souffrance des mères correspond la souffrance des enfants qui grandissent dans des familles transnationales, souffrant de leur solitude, de leur insécurité et de leur vulnérabilité. Privés de l'intimité des interactions quotidiennes, ils cherchent à comprendre les motifs à l'origine de la décision de leurs mères de les élever à distance et ne semblent pas convaincus que la tendresse et les soins psychologiques puissent être entièrement fournis par les réseaux de parenté, par le soutien financier des mères et par les appels téléphoniques hebdomadaires.

Parreñas met en cause l'influence des attentes produites socialement par la maternité traditionnelle, qui aggravent les conflits intergénérationnels des familles transnationales. En définissant les attentes et les désirs, les enfants suivent la distribution des rôles de genre à l'intérieur de la famille. Cette construction idéologique n'influe pas seulement sur leurs opinions mais aussi sur les sentiments et les émotions qui concernent la séparation familiale.

De façon surprenante, Parreñas ne considère pas le regroupement familial comme une option possible, bien qu'elle ait aussi interviewé en Italie des enfants qui avaient rejoint leur mère. Les cas de regroupement dont elle parle sont à leur tour des expériences de souffrance et elle voit dans les protagonistes plus des victimes des évènements que des acteurs capables d'initiative. Quant au lien entre souffrance affective et valeurs traditionnelles, l'auteur semble ne pas se rendre compte que l'emphase donnée à ce rapport affaiblit la thèse fondamentale d'une injustice à l'échelle globale qui pèse sur les enfants des familles transnationales : si le problème était seulement ou essentiellement idéologique, il suffirait pour remettre les choses en place, en s'appuyant sur des outils de communication appropriés, de remplacer la conception désuète que ces jeunes ont de la famille par une idéologie plus adaptée au contexte actuel de care drain, capable de convaincre par exemple les enfants qu'ils peuvent grandir tout aussi bien sans leurs parents auprès d'eux. Mais ainsi la demande de politiques plus généreuses au sujet des regroupements perdrait du terrain, au grand avantage des pays qui reçoivent et des familles qui drainent des ressources de soin à partir des pays les plus pauvres. 
Dans Children of global migration, Parreñas, en plus de prêter une attention à l'option des regroupements familiaux et aux difficultés liées à la réglementation que rencontrent les familles dans un pays ayant des politiques de migration en apparence libérales comme les États-Unis, insiste sur les attentes culturelles qui alourdissent le fardeau des mères transnationales, en les poussant à surcompenser leur éloignement par l'intensification des soins à distance. De même, les institutions, qui œuvrent pour promouvoir des réglementations plus favorables aux regroupements familiaux, sont critiquées, puisqu'elles finissent par confirmer les organisations familiales traditionnelles, en idéalisant la famille nucléaire (2005 : 54). Bien plus clairement que dans son ouvrage précédent, la solution du problème est culturelle et idéologique. L'auteure défend l'idée que les jeunes, les pères, les institutions s'adaptent à la situation en élaborant de nouveaux modèles familiaux dans lesquels on puisse faire abstraction de la présence des mères et fournir d'une autre façon les soins et la sécurité affective dont les enfants ont besoin.

Le livre au contraire montre une certaine discontinuité d'avec le précédent lorsque, dans le but de s'opposer à la stigmatisation des femmes émigrantes en tant que présumées responsables de la désagrégation des familles, de la souffrance affective et du malaise psychologique des enfants laissés au pays, il s'aligne sur le courant de littérature qui met en relief les processus de réorganisation du système de soins parentaux dans les familles transnationales. Grâce à la mobilisation des réseaux de parenté, des filles plus âgées, mais aussi de femmes salariées plus pauvres, ainsi qu'à travers l'effort de garder vivante une relation familiale malgré les distances, par toutes sortes de pratiques et rituels : appels téléphoniques hebdomadaires à des heures précises, messages SMS quotidiens à l'heure du réveil, conseils à distance dans des domaines divers, allant des problèmes de santé jusqu'au menu de la semaine, de nombreuses mères parviendraient à confirmer leur propre rôle et à garder une relation affective et intime, malgré l'éloignement, avec leurs propres enfants.

\section{PRENDRE SOIN À DISTANCE}

En tenant compte de ces dernières considérations, une piste de recherche intéressante, consiste à étudier les ressources et les stratégies que les familles internationales mettent en œuvre pour essayer de remédier à la séparation physique et garder vivaces les rapports affectifs malgré les distances.

Ici apparaît l'importance du rôle des caretaker, des personnes qui prennent soin des enfants à la place de leur mère, comme figure centrale dans la médiation du rapport entre parents à l'étranger et enfants restés au pays. Le caretaker préféré est la grand-mère maternelle et lorsqu'il faut recourir à d'autres personnes de la parenté ou à des personnes salariées les craintes et les doutes sont plus importants, concernant notamment l'emploi de l'argent et le type de rapport instauré avec les enfants (Hondagneu-Sotelo et Avila, 1997).

Les envois d'argent prennent alors une valeur qui dépasse leur signification économique, en symbolisant la persistance du lien affectif et de la responsabilité 
parentale qui se détache du plan des relations quotidiennes, de la présence physique et des soins matériels pour se situer au niveau d'une sollicitude qui conduit à considérer le départ comme le moyen nécessaire pour assurer de meilleures conditions de vie. Dans cette perspective, les envois d'argent donnent sens à l'émigration (Aranda, 2003 : 621). Les cadeaux envoyés au pays sont peut-être encore davantage chargés de résonances symboliques. Tantôt inutiles, presque toujours très onéreux, les cadeaux - comme le démontre une longue tradition d'études anthropologiques — symbolisent la personne absente. Ils transmettent son affection, certifient son effort pour connaître les goûts et les exigences de celui qui est resté au pays, témoignent du temps investi pour trouver et expédier l'objet offert ; d'autant plus quand il s'agit de cadeaux d'une mère pour ses enfants.

Lettres et photographies sont depuis plus d'un siècle un autre moyen communément utilisé pour être réciproquement présents, pour manifester l'empressement et l'implication dans la vie des êtres chers, pour garder vivant le lien affectif compromis par la distance. Le téléphone aussi remplit une fonction essentielle dans la réécriture des relations familiales des familles transnationales. Jusqu'au début des années 1990, les migrants, selon une étude internationale (Wilding, 2006), préféraient communiquer régulièrement par lettre car le téléphone était considéré comme moins fiable et plus coûteux. Mais, depuis le milieu de la décennie, le modèle de communication a changé, grâce notamment à la baisse des coûts, si bien que, pour citer Benítez « l'utilisation des téléphones portables fournit de nouvelles possibilités de mobilité dans le temps et dans l'espace et d'intégration sociale dans la vie de tous les jours » (2006 : 191). D'autres chercheurs parlent même avec emphase de «mort de la distance » (Cairncross, 1997). À son tour, Diminescu (2005) a tracé la nouvelle figure du «migrant connecté », acteur d'une « culture du lien » et protagoniste d'une « installation relationnelle dans la mobilité ».

Cartes prépayées et téléphones portables se généralisent dans le monde entier, grâce notamment aux exigences de communication des familles transnationales. Sur le plan technique, plusieurs pays et régions du Tiers-Monde sont en passe d'entrer directement dans le monde de la communication « sans fil ». Sur le plan micro-social, le contact personnel, en temps réel, permis par la téléphonie à bas coût, serait en train de transformer la vie quotidienne des migrants et de leur famille : les discussions qui autrefois se déroulaient autour de la table, concernant l'achat d'un appareil électroménager, le comportement des adolescents ou les personnes âgées dont il faut prendre soin, peuvent à présent se faire par téléphone. Ce moyen permet aux personnes de la famille, dispersées par l'émigration de garder un sens de la collectivité, de se considérer et de fonctionner comme des familles (Vertovec, 2004b : 222) ${ }^{9}$.

9 Quelques données, bien qu'anciennes, peuvent donner une idée du recours au téléphone : le volume global des appels a progressé de 12,7 milliards d'appels/minute en 1982 à 42,7 en 1992, atteignant 154 milliards d'appels en 2001. Pour le détail, entre 1995 et 2001 les appels de l'Allemagne vers la Turquie ont augmenté de $54 \%$; du Royaume-Uni vers l'Inde de $439 \%$, vers le Pakistan de $390 \%$; des États-Unis vers le Mexique de $171 \%$, des Philippines de $452 \%$ (Vertovec, 2004b : 219). 
Si les lettres permettent, du moins en théorie, l'expression d'un discours plus personnel et approfondi, le téléphone offre la possibilité d'une interaction directe et immédiate dans laquelle on peut transmettre par la voix des sentiments et des émotions. Ainsi il est possible aux parents d'être en communication avec leurs enfants en des moments particulièrement chargés de signification (anniversaires, jours de fête, rentrée scolaire, examens, ...).

Des outils de communication plus avancés, comme le message électronique et la webcam, bien que pour le moment accessible à peu de gens (Banfi et Boccagni, 2007), se développent parmi les migrants ; moyens alternatifs pour ressouder les liens entre les membres des familles transnationales. Selon Wilding, depuis la fin des années 1990, internet (utilisé par environ un tiers des familles interviewées dans sa recherche) a permis une intensification de la communication, à tel point que «l'introduction du courrier électronique a transformé la famille transnationale ». Le premier avantage est qu'il contribue à donner une perception d'intime connectivité (2006: 138). Ces dernières années, le recours diffus aux messages SMS s'est ajouté à la liste des outils de la communication à distance, sans pour autant faire disparaître la communication à travers les lettres. Wilding, cependant, observe qu'à côté des contacts plus fréquents et d'une augmentation des sentiments de proximité dans la vie quotidienne, il arrive que la communication régulière intensifie, au lieu de diminuer, la perception de la distance : « l'impossibilité d'avoir un contact face à face est parfois plus poignante alors que la communication à longue distance fait percevoir la relation plus intimement connectée » (2006 : 138-139). D’autre part, selon Benítez (2006), une différenciation apparaîtrait entre les réseaux familiaux transnationaux mieux dotés économiquement, techniquement et culturellement, en mesure d'utiliser des moyens de communication plus avancés, et ceux qui ne possèdent pas ces moyens. Alors que Parreñas (2005) met en relief la possibilité d'être proche grâce au téléphone et à ses dérivés, en faisant ressentir la présence des parents aux moments les plus importants, d'autres analyses ont mis l'accent sur la réticence et l'insécurité de la communication à distance : dans différentes situations critiques, de part et d'autre, peut prévaloir le souci de ne pas inquiéter les conjoints qui ne seraient de toute façon d'aucune aide ; ou alors on se sent mal à l'aise pour parler des problèmes au téléphone ou par courrier électronique.De plus, avec le temps et la prolongation de l'éloignement, les « choses à se dire » auraient tendance à diminuer et la communication ne pourrait se réduire qu'à des formules brèves et stéréotypées.

La séparation forcée entre les membres de la famille amène donc au développement de différentes stratégies, à travers lesquelles les familles transnationales s'efforcent de maintenir les liens en dépit de la séparation physique. Selon la conceptualisation souvent citée de Bryceson et Vuorela (2002), une première stratégie est nommée frontiering et désigne les moyens utilisés par les membres des familles transnationales pour alimenter des rapports familiaux et des liens affectifs à travers les frontières, dans des cas où les rapports de parenté sont relativement dispersés. Les frontières politiques ne sont alors plus considérées comme des barrières rigides et insurmontables, mais elles deviennent des espaces traversés de différentes façons par les rapports familiaux. 
La deuxième stratégie, qu'ils nomment relativizing, fait référence aux différentes façons choisies par les individus pour établir, maintenir ou interrompre leurs rapports avec les autres membres de leur famille. Dans les familles transnationales les rôles familiaux traditionnels de père, mère, fils, frère, sœur, ainsi que ceux des membres de la famille élargie, oncles, tantes, cousins, se redéfinissent, se substituent ou se suppriment. Des liens se perdent, d'autres s'acquièrent ou se renforcent, par exemple en inventant des formes de parenté fictive (comme l'appellation d' « oncle » attribuée à une connaissance influente et bienfaisante) ou en établissant des rapports de parrainage. Face à une expérience d'appauvrissement des contacts avec les personnes auxquelles le migrant est uni, il éprouve le besoin d'expliquer pourquoi et comment des membres de la parenté proche ou lointaine, ou une partie d'entre eux, font cependant partie de sa propre famille. Ils repensent et recodifient les liens affectivement significatifs en réécrivant d'une certaine façon leur propre histoire familiale ${ }^{10}$.

Mais une grande partie de la littérature sur les familles transnationales place au centre de ses observations une troisième stratégie qui reflète la principale préoccupation des familles migrantes avec des enfants en bas âge et qui peut être définie comme le soin à distance : la majeure partie des investissements, des relations, de la sollicitude même envers la famille étendue, sont liés à la nécessité d'assurer aux enfants laissés au pays un contexte de soins matériels et de protection affective. La gestion des relations familiales à travers les frontières et la géométrie variable des rapports avec le réseau parental s'expliquent par le besoin de privilégier les liens avec les conjoints capables de prendre en charge l'éducation des enfants. La relation mèreenfants, dans ses différentes déclinaisons, est le noyau dur de l'organisation complexe des familles transnationales.

L'omission de cet aspect porte au contraire Bryceson et Vuorela à extraire de leur analyse une conclusion discutable : la famille, tout comme la nation, ne serait autre qu'une « communauté imaginée », donc une construction idéologique et abstraite. Il me semble par contre que la comparaison famille/nation démontre justement le contraire : l'appareil politique et idéologique ayant construit et consolidé les nations a fait en sorte que les individus se sentent d'une certaine façon affectivement solidaires avec d'autres qu'ils ne connaissent pas grâce à des processus d'identification à cette nation. Par contre ils ne sont pas nombreux à ressentir l'obligation d'adopter des comportements effectifs, engageants et personnellement coûteux, pour rendre opérationnel ce sens de la solidarité collective. Des pratiques comme payer des impôts, ou, jusqu'à récemment, l'accomplissement des obligations militaires, nécessitent un appareil contraignant sans lequel elles ne seraient pas respectées.

10 Une analyse spécifique (Thompson et Bauer, 2005 : 16-17) a identifié quatre types d'aide mutuelle entre les familles transnationales, en élargissant le regard au-delà de la prise en charge des enfants, et en considérant les deux versants des échanges généreux des processus migratoires : assistance pratique dans la migration (paiement des billets, premier logement, aide pour la recherche d'un emploi) ; envoi d'argent aux parents du pays d'origine (sous forme d'argent ou de cadeaux) ; aide pour la prise en charge des enfants et permettre à une jeune mère d'émigrer et de travailler ; prise en charge et assistance des personnes âgées. 
Au contraire la grande majorité des migrants (et plus particulièrement les mères) est actrice d'incessants et douloureux efforts pour garder vivants les liens familiaux, pour assurer les frais nécessaires à la vie des enfants restés au pays, pour regrouper leur famille dès que l'occasion se présente. Ces efforts s'adressent à des personnes concrètes et connues de façon intime, avec lesquelles il y a des liens biologiques et sociaux. Ils sont entrepris volontairement et soutenus par des obligations morales intériorisées : les liens avec les enfants restent profonds, contraignants, denses même dans des circonstances défavorables alors qu'il n'existe pas d'institutions en mesure de les imposer. Les parents et surtout les mères continuent à se sentir responsables de leurs enfants même si la séparation physique se prolonge. Réécrire les relations familiales, avec le renforcement de certains liens et l'atténuation ou la suppression de certains autres, se situe sur un plan secondaire par rapport à la prise en charge des enfants.

Affirmer que la famille est une institution dynamique, connaissant des adaptations, des réélaborations, des négociations et des échecs, ne me semble pas conduire à la cataloguer comme une construction sociale artificieuse et «imaginée ». Si l'on prend au sérieux, en suivant l'invitation de Semi (2007) les accounts des acteurs (ou bien les interprétations qu'ils donnent de la réalité) et leurs pratiques sociales (practices) (Baumann, 1999), on ne peut méconnaître la place centrale des liens familiaux et surtout du rapport avec les enfants, dans les récits et dans la myriade d'efforts que les mêmes migrantes accomplissent pour essayer de garder, malgré tout, une présence active dans la vie des proches. Le discours constructionniste de Bryceson et Vuorela, s'il est mené avec cohérence jusqu'aux conséquences extrêmes, risque de comporter un effet de diminution de la charge d'injustice inhérente à la stratification internationale des opportunités de soin et à la demande politique de plus de liberté à l'égard des regroupements familiaux : si la famille était vraiment juste une «communauté imaginée », les immigrés pourraient, tout compte fait, en imaginer une autre ou bien l'imaginer différemment, tandis qu'ils auraient moins de droits pour insister, s'ils le désirent, sur le droit à vivre ensemble à celle qu'ils continuent à percevoir comme la leur.

Quant au nœud du rapport entre mères transnationales et les enfants restés au pays, et plus particulièrement à la possibilité de grandir sereinement sans être assistés de près par la présence maternelle, les recherches (peu nombreuses) qui ont tenté $\mathrm{d}$ 'affronter le thème en dehors des situations pathologiques (comme ceux traités par les services sociaux) mettent l'accent sur la qualité des différentes relations qui exercent un rôle influent dans le processus d'arrangement : le rapport entre parents et enfants avant le départ, le rapport avec le caretaker et les enfants, et le triangle formé par le caretaker, les enfants et les parents. Des problèmes surgissent lorsque la mère se sent menacée par le rôle assumé par le caretaker, ou s'il culpabilise et délégitime la mère, ou est déprimé à cause de son départ. Quand, par contre, les figures adultes arrivent à collaborer de façon efficace, les crises sont moins fréquentes et la probabilité de créer des adaptations plus efficaces augmente selon Suarez-Orozco, Todorova et Louie. De plus, malgré l'adversité qui découle de la séparation forcée, les enfants souvent développent une importante résilience. Si les enfants sont bien préparés, si la séparation est perçue comme temporaire et nécessaire, entreprise pour le bien de la familles, elle est alors mieux gérable en comparaison aux situations où les enfants se sentent abandonnés (2002 : 640). 
En plaçant au centre l'image des mères à distance, l'histoire des familles transnationales confirme le caractère des migrations comme phénomène social « gendered » c'est-à-dire influencé par les rapports de genre, ainsi que gendering, c'est-à-dire édificateur de nouveaux rapports de couple (Hondagneu-Sotelo, 1992). On peut souligner que la configuration des relations de genre dans les familles transnationales prend des aspects particuliers, porteurs en même temps de souffrance et d'innovation. Lors de la séparation physique, les traditions patriarcales s'altèrent et dans de nombreux cas les femmes deviennent plus compétentes et autonomes, par exemple dans la gestion de l'argent. L'autorité du mari diminue et l'influence de la femme augmente. La répartition même du travail domestique tend à être revue, puisque les hommes peuvent être amenés à apprendre des tâches traditionnellement féminines, telles que cuisiner, laver, repasser, veiller au rangement de la maison, et que les femmes, lorsqu'elles restent au pays, peuvent avoir à exercer des prérogatives masculines comme la conduite de véhicules, l'embauche de travailleurs agricoles, l'achat de semences et la vente des récoltes, comme l'a noté Pribilsky (2003) dans un contexte rural en Équateur. Les femmes, dans le cas étudié, développent aussi des relations plus intenses avec d'autres femmes, en échangeant des ressources et en mettant en place des formes d'aide réciproque. Parfois elles assument aussi des rôles publics dans les communautés locales, rôles laissés vacants par le départ des maris.

Quelque chose de semblable survient aussi lorsque les femmes émigrent seules : non seulement elles doivent devenir autonomes dans la gestion de l'argent et dans leurs déplacements, mais souvent elles font naître des circuits d'échange et d'aide mutuelle, gèrent l'arrivée et l'intégration de parents et de compatriotes, mettent en place des formes de prêts par roulement, s'impliquent dans le milieu associatif, accédant à des rôles de leadership (Ambrosini et Abbatecola, 2004).

À ce sujet on a souvent noté que, pour certaines émigrations à très forte prévalence féminine comme celle des Philippins, une vie associative intense, favorisée par le rapport avec les institutions catholiques (et dans une moindre mesure, évangéliques), semble avoir eu un rôle dans un début de diversification des emplois : outre le service domestique on les retrouve parmi les salariées des entreprises de nettoyage, les gardiennes d'immeubles etc. Il se construit ainsi des réseaux migratoires distincts par le genre sur lesquels seules les femmes peuvent s'appuyer pour contrebalancer le pouvoir des maris.

Cependant les études à ce sujet convergent pour montrer aussi l'autre aspect de l'autonomie et de l'empowerment obtenus avec le détachement de la famille : les femmes émigrantes ressentent de façon plus aiguë la frustration dérivant des contraintes liées à un exercice que l'on pense approprié de leur responsabilité de soin, surtout lorsqu'elles doivent affronter des moments critiques de la biographie familiale, tels que les divorces, les naissances, les maladies, les décès (Aranda, 2003). Exercer à la fois un rôle professionnel et un rôle familial traditionnel, déclenche des tensions émotives et des attentes irréelles, qui produisent des sentiments d'inadéquation et de dépression (Dion et Dion, 2001). 
L'éloignement du réseau familial élargi a une incidence tant sur les aspects pratiques que sur la dimension affective des activités de soin qu'on leur demande. En même temps, comme nous l'avons vu, ce sont habituellement les réseaux familiaux du côté féminin du couple qui compensent l'absence des mères en leur permettant d'assurer à distance les devoirs parentaux attendus. Il en est souvent de même, non sans tensions et sans ambivalences, lorsqu'il faut s'occuper de parents âgés (Baldassar, 2007).

Ce sont toujours les femmes aussi, qui sont les plus actives dans la communication à distance avec la famille, surtout par courrier ou par téléphone (Wilding, 2006), en développant, ce qui, il y a vingt ans, di Leonardo (1987) avait appelé kinwork, c'est-à-dire la tâche de maintenir les relations familiales.

\section{UNE TYPOLOGIE DES FAMILLES TRANSNATIONALES}

Une enquête menée en Roumanie et en Ukraine (Castagnone et al., 2007 ; Chaloff et Piperno, 2007) a confirmé que, suite au départ de la mère, la famille élargie se réorganise, en mobilisant d'autres figures du réseau de la parenté (grands-mères, tantes, etc.) pour compenser cette absence. Si cela ne suffit pas, une sorte de marché privé de la prise en charge et des soins familiaux se met en place, dans lequel entrent en action des aides à domicile, des auxiliaires parentales, des enseignants, des tuteurs, des collèges religieux, des adultes qui viennent vivre avec le mineur et exercent un rôle d'assistance/surveillance en échange de l'hébergement. Des écoles et des ONG agissent parfois pour promouvoir des initiatives de contrôle et de soutien aux enfants restés au pays. Grâce aux stratégies mises en place par les familles transnationales, l'impact du care drain est amoindri, et, selon les auteurs, les abandons sont exceptionnels.

Malgré cette mobilisation articulée, il subsiste cependant la sensation d'un manque (un care shortage), d'un réseau sous effort, d'une précarité diffuse, dus également au fait que souvent l'émigration de la mère survient dans des contextes familiaux déjà éprouvés par des difficultés et des crises de différentes sortes : les solutions imaginées ne résistent pas toujours dans le temps, la différence d'âge entre grands-parents et petits enfants peut se révéler trop profonde, ou bien le déplacement d'une ville vers un village peut causer des problèmes; ainsi un certain nombre d'enfants se voient dans l'obligation de changer à plusieurs reprises de logement et d'adultes référents.

Dans d'autres cas, le fait de confier les enfants à des parents ou à des voisins qui assument le rôle de tuteurs entraîne une relation d'affection et d'intimité souvent difficile, de sorte que les enfants laissés au pays se retrouvent dans un état intermédiaire entre être hébergé et être de véritable membre de la famille dans laquelle ils vivent, condition qui rappelle la familiarisation ambiguë du rôle des mères dans la famille où elles travaillent. 
La majeure partie des mineurs, cependant, arrive à réagir au sentiment d'abandon. Bien qu'ils souffrent manifestement de la séparation, les enfants des mères émigrées cherchent à ne pas donner d'eux et de leur famille une image négative de sujets désavantagés, mais bien au contraire mettent l'accent sur la recherche d'un équilibre affectif, dans le cadre d'une nouvelle vie.

Concernant les deuxièmes générations philippines, Zanfrini et Asis (2006) fournissent des éléments pour l'analyse des familles transnationales, en se référant à des enquêtes réalisées par le Scalabrini Migration Center. Il en ressort notamment que la plupart des enfants d'émigrées déclarent être assistés en premier lieu par leurs mères, même quand celles-ci sont à l'étranger. De plus, les garçons restés au pays ont des résultats scolaires meilleurs que ceux des autres jeunes de leur âge, parce qu'ils fréquentent des écoles privées mieux équipées et dont les classes sont moins nombreuses. Pour ce qui est de la mobilité, la trajectoire de ces enfants ne suit pas toujours le tracé conventionnel (naissance dans le pays d'origine des parents puis regroupement), mais on constate aussi, par exemple, des naissances en Italie, suivies de transferts aux Philippines pour être mieux suivis dans leur croissance par les grandsparents ou d'autres parents encore, plus disponibles. Ainsi les parents ne souhaitent pas, dans ces cas, un regroupement avec leurs enfants en Italie, car ils craignent que les énormes investissements faits pour leur éducation ne soient sous-utilisés. Quand le regroupement a lieu, il est souvent justifié par l'échec scolaire ou la perte de motivation envers l'instruction. Les enfants à leur tour, souvent poussés vers l'émigration, ne considèrent pas l'Italie comme une première destination, même si les parents y sont installés depuis des années, mais pensent à la péninsule comme une destination de vacances plutôt qu'à un endroit où ils pourront trouver un travail satisfaisant. Dans l'ensemble se dessine un tableau où l'émigration ne s'oppose pas à la cohésion familiale, mais représente plutôt un choix familial partagé, avec pour but la promotion sociale de la famille elle-même, et plus particulièrement celle des enfants, choix que les enfants semblent comprendre et accepter, tout en déclarant, bien sûr, préférer que la famille reste unie ou au moins que ce soient les pères et non les mères qui partent.

Les résultats des recherches ainsi rappelées suggèrent donc que la réalité sociale des familles transnationales est très diverse. Ni les visions catastrophiques, ni celles qui tendent à minimiser les problèmes ne peuvent rendre compte convenablement de sa complexité.

Notre recherche sur les aides à domicile (Ambrosini et Cominelli, 2005) avait proposé une typologie des familles transnationales, où entraient en jeu l'âge, la condition familiale, la distance, le projet de migration et les ressources personnelles, identifiant ainsi des profils et des trajectoires différents :

- un profil explorateur, de femmes très jeunes, sans charges familiales, arrivées en Italie et employées de façon plutôt occasionnelle, qui cherchent à sonder les opportunités offertes par le contexte, à reprendre autant que possible leurs études et à participer aux formes de sociabilité de leurs camarades italiens ;

- un profil utilitariste, de femmes plus âgées (à partir de 45 ans environ) venant surtout d'Europe de l'Est, qui ont laissé au pays des enfants déjà grands qui 
dépendent de leurs remises mais qui n'ont pas l'intention de les rejoindre. Elles ne cherchent pas à s'établir, mais pratiquent des formes de va-et-vient entre l'Italie et leur pays, travaillent et épargnent le plus possible, projettent de rentrer définitivement dans leurs pays dans un petit nombre d'années ;

- un profil familialiste, plus proche de l'image des mères transnationales diffusée par la littérature : des femmes, jeunes adultes, en provenance surtout d'Amérique latine, ayant laissé au pays des enfants en bas âge, qui aspirent au regroupement familial et dont la perspective est de se mettre en règle, trouver ensuite un emploi d'assistante non logée et un logement autonome pour recomposer l'unité familiale ou, au moins, vivre avec les enfants ;

- un profil promotionnel enfin qui concerne aussi des femmes jeunes adultes venant de pays divers, ayant de hauts niveaux d'instruction, des expériences professionnelles significatives dans leur pays, des aspirations à améliorer leur propre statut, et qui vivent avec un fort sentiment de frustation leur situation professionnelle actuelle. Lorsqu'elles ont des enfants, leur responsabilité parentale - qui implique l'envoi régulier d'argent - risque d'inhiber leurs investissements dans la formation et leur recherche d'un emploi au niveau de leurs aptitudes.

Les recherches italiennes récentes (Castagnone et al., 2007 ; Banfi et Boccagni, 2007) ont progressé dans la même direction : saisir les différences et les spécificités des flux migratoires qui permettent le welfare informel, en subissant des processus de care drain dans le cadre familial. Ces flux présentent des configurations variables. Dans la recherche CESPI-FIERI, les caractéristiques des Roumaines sont différentes de celles des Ukrainiennes. Leur âge moyen est plus bas, elles doivent subvenir aux besoins d'enfants plus jeunes, elles disposent enfin, depuis l'entrée de leur pays dans l'Union Européenne, de larges possibilités d'entrée et de circulation. Dans l'étude de Banfi et Boccagni, nous trouvons un cas de circulation migratoire, dans l'immigration des femmes polonaises, elles aussi citoyennes de l'Union Européenne, avec de fréquents allers-retours et de visites de leurs familles en Italie, et un projet de retour plutôt que de regroupement familial. Les Ukrainiennes ont un profil différent. Les migrantes sont des femmes d'âge mûr, souvent de jeunes grands-mères, chargées de répondre aux demandes d'argent de plusieurs générations, mais n'envisageant pas le regroupement familial en Italie. Elles ont comme spécificité de vivre leur émigration dans des conditions matérielles très dures, mais en disposant d'une liberté qui ne serait pas concevable dans leur pays. Les Équatoriennes sont plus proches du modèle des mères à distance, où l'éloignement géographique empêche des rapports fréquents et génère des privations affectives. Elles sont écartelées entre la perspective du regroupement familial et celle de la rupture du mariage.

Il n'existe donc pas qu'un seul type de famille transnationale, mais une pluralité de stratégies d'adaptation à la séparation et de réponses au care drain, dans lesquelles des éléments biographiques comme l'âge, des facteurs objectifs comme les distances, des ressources politiques comme la citoyenneté, des éléments particuliers comme la composition et la solidité du réseau familial élargi, définissent le périmètre où s'exercent les efforts des familles séparées pour rester en contact et subvenir aux nécessités des enfants. 
En substance, concluent Banfi et Boccagni, la vie familiale transnationale s'articule selon des modèles divers, alors que dans le champ des relations entre générations (et surtout dans la dyade mère-enfants) le scénario global montre des caractéristiques beaucoup plus convergentes. La sollicitude des parents (et de façon croissante, celle des mères migrantes) dans le soutien aux enfants laissés au pays, et pour de nombreux aspects, par le fait d'en prendre soin, à distance, justifie ainsi l'emploi de l'étiquette «familles transnationales ».

En partant de ces considérations et aussi des résultats des recherches citées, on peut arriver à l'ébauche d'une typologie des familles transnationales. Il convient de préciser qu'il s'agit d'un chantier ouvert, se référant à un champ de recherches en grande partie nouveau et continuellement en évolution. Des dispositions légales, telles les mesures de régularisation des immigrés irréguliers ou les élargissements de l'Union Européenne vers l'Est, ont notablement modifié, en peu d'années, non seulement le statut juridique, mais aussi la possibilité de mouvement et de permanence, de changement d'emploi et de regroupement familial, pour de nombreuses mères migrantes. La situation, sans aucun doute, changera encore. Par exemple, comme cela se passe en général pour tous les flux migratoires, alors qu'aujourd'hui la migration temporaire l'emporte, on peut s'attendre dans un futur proche à des installations durables. Dans le cas des nouveaux pays membres de l'Union Européenne, l'expérience du passé fait penser à un déclin prévisible de la tendance à émigrer et à un prochain retour d'une partie des émigrés. Avec ces précautions, on peut essayer d'organiser sous forme typologique les résultats des recherches citées au sujet des familles transnationales (tableau).

Tableau 1 : Typologie des familles transnationales (cas italien)

\begin{tabular}{|l|l|l|l|}
\hline & $\begin{array}{c}\text { Familles } \\
\text { transnationales } \\
\text { mouvantes }\end{array}$ & $\begin{array}{c}\text { Familles } \\
\text { transnationales } \\
\text { intergénérationnelles }\end{array}$ & $\begin{array}{c}\text { Familles } \\
\text { transnationales } \\
\text { centrées sur les enfants }\end{array}$ \\
\hline Protagonistes & $\begin{array}{l}\text { Mères adultes et } \\
\text { d'âge mûr }\end{array}$ & $\begin{array}{l}\text { Mères d'âge mûr, } \\
\text { jeunes grands-mères, }\end{array}$ & Jeunes mères \\
\hline Personnes à charge & $\begin{array}{l}\text { Enfants d'âge } \\
\text { différent }\end{array}$ & $\begin{array}{l}\text { Enfants déjà grands et } \\
\text { souvent des petits- } \\
\text { enfants }\end{array}$ & Enfants en bas âge \\
\hline Provenance & $\begin{array}{l}\text { Nouveaux pays UE } \\
\text { (par ex. Pologne, } \\
\text { Roumanie }\end{array}$ & $\begin{array}{l}\text { Europe de l'Est non } \\
\text { UE (par ex. Ukraine, } \\
\text { Moldavie) }\end{array}$ & $\begin{array}{l}\text { Surtout des pays extra- } \\
\text { européens } \\
\text { (Amérique Latine, } \\
\text { Afrique, Philippines) }\end{array}$ \\
\hline Retours au pays & Fréquents & Assez fréquents & $\begin{array}{l}\text { Rares (problème des } \\
\text { distances et des coûts) }\end{array}$ \\
\hline $\begin{array}{l}\text { Regroupements } \\
\text { familiaux }\end{array}$ & Non souhaités & Non prévus & $\begin{array}{l}\text { Souhaités ou } \\
\text { néanmoins pratiqués }\end{array}$ \\
\hline Projet migratoire & Alternance & Retour & $\begin{array}{l}\text { Orienté vers } \\
\text { l'établissement en Italie } \\
\text { (Amérique Latine); } \\
\text { investissement dans les } \\
\text { études des enfants } \\
\text { (Philippines) }\end{array}$ \\
\hline
\end{tabular}




\section{SÉPARATION, DISTANCE, REGROUPEMENT : LES TROIS FAMILLES DES MIGRANTS}

Il s'agit à présent de comprendre la dimension dynamique des migrations familiales, dans laquelle s'inscrit le facteur transnational. En réalité, peu de familles arrivent déjà formées et au complet dans les sociétés d'accueil et quand cela arrive il s'agit de migrants hautement qualifiés, d'entrepreneurs, de cadres supérieurs, de chercheurs qui se déplacent à l'étranger pour quelques années ou définitivement — en emmenant avec eux le noyau familial au complet — ; ou alors, dans le cas contraire, de réfugiés qui fuient les guerres et les persécutions. Le plus souvent la migration familiale est un processus à plusieurs étapes : la famille qui vit ensemble dans le pays d'origine doit affronter l'épreuve de la séparation, lorsque celui (ou celle) qui émigre a le plus de possibilités pour franchir les frontières et trouver un travail ; puis arrive le moment de l'éloignement et des liens affectifs à distance ; enfin a lieu le moment du regroupement et de la recomposition du noyau soit avec le retour au pays, soit, plus fréquemment, par le déplacement de la famille dans les sociétés d'accueil dès que le premier émigrant a réussi à atteindre un niveau d'intégration acceptable sur le plan économique et celui du logement ${ }^{11}$. C'est la dynamique que certains ont décrit en termes des «trois familles» des migrants (Esparragoza, 2003). En tout cas, « la migration implique des processus de fragmentation et de regroupement de l'unité familiale qui habituellement provoquent des changements structurels substantiels dans le fonctionnement de la famille » (Torrealba Orellana, 1989, cité par Lagomarsino, $2006: 185)$.

Tout d'abord, la décision d'émigrer se place dans un espace se situant entre d'une part le choix partagé par les époux et soutenu par la famille élargie avec comme but l'augmentation du bien-être du groupe, d'autre part le choix individuel de rupture contre l'avis et les intérêts du groupe familial. Dans le cas de femmes qui partent seules, l'émigration peut aussi être le résultat d'une crise matrimoniale, puisqu'elle offre une opportunité socialement légitime pour sortir de situations considérées comme insupportables ${ }^{12}$.

La famille regroupée est bien différente de celle laissée au pays des années auparavant, mais aussi de celle imaginée pendant la séparation forcée, ou retrouvée pendant de brefs retours au pays pour les vacances. Non seulement les enfants ont grandi, mais le migrant a changé, comme son conjoint resté au pays. L'équilibre et les rapports internes et externes au couple se sont transformés. Vivre à nouveau ensemble

11 En Italie, les demandes de regroupement présentés en 2007 ont été d'environ 85 000, soit $73 \%$ de plus qu'en 2006 où elles avaient été au nombre de 49 000. La moitié des demandes concernait les conjoints, presque autant les enfants, plus rarement les parents. 82330 visas ont été accordés par les consulats italiens à l'étranger en 2006.

12 Par exemple aux Philippines, cette situation est définie comme un « divorce philippin ». 
dans une société différente de celle dans laquelle les époux s'étaient rencontrés et avaient construit le lien familial, dans un contexte où seulement l'un d'eux connaît un peu le pays, la langue, le fonctionnement des institutions et les pratiques sociales quotidiennes, où le soutien du réseau de la parenté fait défaut, où l'insertion scolaire et sociale est délicate, tout cela est une nouvelle source de stress qui met à l'épreuve la solidité du couple (Aranda, 2003).

Pour les enfants, d'autres problèmes surgissent. Tout d'abord ils connaissent le plus souvent une baisse de leurs conditions matérielles de vie et de consommation, passant du statut de jeunes nantis ayant la possibilité de dépenser davantage que la moyenne des jeunes de leur âge grâce à l'argent envoyé par leurs parents au statut de jeunes de classe populaire, avec des revenus familiaux modestes et des conditions de logement peu confortables. De plus, ils sont fréquemment confrontés dans leur nouveau contexte de vie à la discrimination et au racisme.

Pour les parents, l'absence prolongée et la perte d'intimité et de connaissance réciproque rendent difficile la légitimation de leur propre autorité et révèlent l'insuffisance de la dimension économique, si vitale au moment de l'éloignement, comme base pour recodifier les liens, lorsque toute la famille se trouve à nouveau sous le même toit, dans un nouveau pays (Hondagneu-Sotelo et Avila, 1997 ; Bonizzoni, 2007).

De plus, la littérature montre que, bien que désireux de rejoindre les parents dans la terre promise de l'émigration, les enfants doivent ensuite faire face à la séparation avec ceux qui ont pris soin d'eux, parfois pendant plusieurs années (SuarezOrozco, Todorova et Louie, 2002), et aussi de leurs groupes des pairs, qui, en l'absence des parents, étaient devenus un point de repère pour les adolescents. Ainsi les regroupements suivis d'échec et les retours au pays ne manquent pas. Des variables comme l'âge, la durée de la séparation, le rôle assumé par le caretaker et son rapport avec les parents, entrent certainement en jeu pour influencer les dynamiques et les résultats du regroupement (Bonizzoni, 2007). De toute façon le lien à distance ne représente pas seulement une parenthèse de séparation forcée, mais bien une phase évolutive qui influence profondément les développements à venir des relations familiales.

Les enfants doivent ensuite tenir compte des attentes des parents souvent ressenties comme opposées, entre l'incitation à acquérir des attitudes et les compétences favorables à la mobilité sociale, et la volonté de transmettre des codes de comportement et des valeurs morales traditionnelles. Cette tension est particulièrement aiguë pour les filles (Dion et Dion, 2001), envers lesquelles le contrôle et les attentes de comportements conformes sont habituellement plus pressants.

Quelques variantes peuvent venir compliquer ces processus : quand la femme est là l'origine du regroupement, inversant les rôles traditionnels (Lagomarsino, 2006). Les maris se retrouvent fréquemment en situation de frustation avec le sentiment de perdre leur rôle, mais aussi leur autorité et leur prestige au sein de la famille. Leur identité de genre s'était construite sur la base de conditions préalables de machisme 
traditionnel, nourri d'idées comme celle de la responsabilité principale du mari dans le travail extra domestique et dans le maintien économique des personnes, de la primauté de son autorité quand il s'agit d'assumer des décisions et d'orienter la vie de la famille, des prérogatives d'une plus grande connaissance et familiarité avec les institutions publiques et la société extérieure, d'une nette division des rôles qui considère la femme comme la gardienne de la sphère domestique, subalterne et dévouée au mari. Toutes ces conditions sont de plus en plus contredites par les transformations sociales, même dans les lieux d'origine et surtout dans les contextes urbains, mais encore bien vivantes culturellement. Le regroupement guidé par les femmes altère profondément ce modèle des rapports de genre : ce sont les épouses qui procurent les ressources économiques pour nourrir la famille, qui organisent le regroupement en décidant le moment et les modalités, qui servent de guide à l'insertion dans la nouvelle société, disposant d'une maîtrise au moins basique de la langue et d'une certaine socialisation dans la société d'accueil. Il est difficile pour les maris de ne pas se sentir dépossédé d'un rôle socialement acceptable, car obligé de dépendre de leurs épouses.

Il faut ajouter que les regroupements inverses se vérifient d'habitude dans les courants migratoires à dominante féminine. Dans la recherche d'emploi, les réseaux construits par les femmes, composés de personnes employées dans les services à domicile et d'assistance, peuvent retrouver une certaine efficacité en proposant des emplois analogues aux hommes. Mais ils ne peuvent que rarement les aider à s'insérer dans des milieux différents. Ce fait aussi contribue à déprimer les maris « regroupés », ce qui rend plus longue et compliquée la recherche d'un emploi acceptable.

Sans vouloir arriver à des conclusions définitives, les issues malheureuses des regroupements de ce genre, avec des problèmes d'alcoolisme, des disputes, des violences domestiques, des ruptures de mariages, sont assez fréquentes. Le scénario peut être encore plus compliqué lorsque se forment de nouvelles unions (Dreby, 2006).

D'autres difficultés apparaissent dans des cas de regroupement partiel surtout ceux qui sont organisés par des femmes séparées ou veuves qui décident de faire venir leurs enfants. Elles les reçoivent parfois dans une nouvelle unité familiale où d'autres enfants sont éventuellement nés (Suarez-Orozco, Todorova et Louie, 2002). Ces situations sont sources de difficultés affectives qui s'ajoutent aux tensions de l'arrivée.

Au contraire, dans d'autres cas, surgissent des processus de redéfinition des rôles : les maris acceptent d'assumer les charges domestiques et de s'occuper des enfants (Lagomarsino, 2006). Les plus âgés, les plus fragiles physiquement et ceux que leur santé rend inaptes aux emplois lourds et précaires comme ceux du bâtiment s'insèrent dans les mêmes emplois que leurs épouses dans le milieu domestique et d'assistance : on commence désormais à trouver aussi un certain nombre d'assistants à domicile, en particulier lorsqu'il s'agit de s'occuper d'hommes âgés et/ou ayant des difficultés pour se déplacer. Après une phase de désorientation, les données de recherche suggèrent que le regroupement puisse conduire à une proximité et une intimité plus grandes (Dreby, 2006 : 640). 


\section{CONCLUSION : LES FAMILLES TRANSNATIONALES ET LA GLOBALISATION PAR LE BAS}

Peu de phénomènes, comme la stratification internationale des opportunités d'assistance et le care drain s'y rattachant, mettent en évidence combien l'immigration est profondément incorporée dans les dynamiques et les besoins de notre société. Pour autant que l'on soit méfiant ou hostile à l'égard de l'immigration étrangère, il semble difficile de soutenir qu'il s'agisse, comme beaucoup continuent à le penser, d'un phénomène essentiellement exogène, provoqué par la pauvreté et le sousdéveloppement, dans lequel sont impliqués malgré eux les pays d'accueil, en tant que lieux d'accostage de foules de désespérés, ou pire, de prédateurs faméliques. Le welfare informel et caché, fondé sur le travail des femmes immigrées, remplit désormais une fonction non substituable dans le fonctionnement quotidien de plusieurs sociétés d'accueil ${ }^{13}$. Par cette voie se brouille également la distinction entre immigrés réguliers, tolérables et aussi utiles, et immigrés irréguliers, dangereux et qu'il faut repousser, puisque ce sont justement les familles des pays d'accueil, dans de très nombreux cas, qui donnent du travail aux femmes (et aussi aux hommes) dépourvus des papiers nécessaires, ce qui favorise de fait l'immigration non autorisée ${ }^{14}$.

C'est ainsi que se pose la question des familles séparées par les distances et les frontières, et surtout des mères transnationales engagées malgré l'éloignement dans une incessante activité à distance, d'abord envers leurs enfants, mais aussi envers d'autres membres de la famille laissés au pays (parents âgés, frères, etc.), ainsi que dans un travail de recodification et d'entretien des liens avec les caretakers, les personnes qui compensent leur absence et avec d'autres composantes de la famille élargie. Deux dimensions cruciales apparaissent. La première concerne la densité affective et existentielle que continue à revêtir le rapport mère-enfants, malgré la séparation. La recherche du bien-être de leurs enfants et de l'amélioration de leur avenir sont à l'origine de leur départ, et aussi des efforts déployés pour maintenir les liens et les assister à distance. La sollicitude du soin reste fondamentale dans le vécu des acteurs, même si elle ne peut s'exercer dans des conditions de proximité physique et de présence quotidienne. Elle ne peut être réduite à un simple échafaudage idéologique, comme le voudraient les positions les plus radicalement constructionnistes.

13 Cela ne signifie pas que l'on doive laisser ce fonctionnement en l'état, sans imaginer des hypothèses d'amélioration des conditions de travail des personnes employées et de la qualité de l'assistance fournie. Une amélioration pourrait avoir lieu si l'on introduisait un « troisième sujet » comme employeur, entre la famille qui demande le service et les personnes disponibles pour le faire. J'ai développé ailleurs cette proposition (Ambrosini, 2005b).

14 En ce qui concerne le cas italien, on peut rappeler que suite au " décret des flux » de 2007, relatif aux entrées autorisées des nouveaux immigrés, 724000 demandes ont été présentées ; parmi lesquelles, environ 400000 concernaient des collaboratrices familiales et des assistantes à domicile demandées par les familles italiennes. Elles étaient presque toujours présentées par des migrants déjà installés et employés en Italie. Les décrets sur les flux, comme l'ont explicitement admis les deux derniers Premier Ministre, sont des canaux déguisés de la régularisation. 
La seconde dimension concerne le tissage des liens transnationaux. Quelle que soit l'évaluation des théories du transnationalisme, il apparaît difficile de nier que sur le plan microsocial la dislocation des unités familiales dont les membres vivent dans des pays différents et surtout l'expérience de la maternité à distance soit le signe d'un accroissement important de la fréquence et de la densité des relations et des échanges qui franchissent les frontières. Les nombreuses possibilités offertes par les technologies de la communication, anciennes et récentes, interviennent pour faciliter de façon nouvelle, les relations à distance entre les familles séparées par l'émigration.

Nous savons cependant que tout cela ne suffit pas. De façon croissante, bien qu'étrangement négligée par la majorité des études sur les familles transnationales, les membres des familles dispersées souhaitent revivre ensemble dans le pays d'immigration. Les oppositions politiques et les difficultés économiques peuvent ralentir ces processus, mais ne réussiront pas à les arrêter, en particulier dans les sociétés libérales de l'Occident. Il en découle trois implications en termes de politiques d'immigration. La première dérive de l'observation de la différenciation des familles transnationales. Si nous ne pouvons nous passer, dans un futur prévisible, du travail de soin et d'assistance des femmes immigrées, on peut se demander s'il ne serait pas préférable d'orienter les politiques de recrutement, quand cela est possible, opportun et souhaité par les intéressés, vers les femmes d'âge mûr, déchargées des responsabilités auprès d'enfants en bas âge, mais souhaitant aider avec les revenus de leur travail les générations plus jeunes, comme cela s'observe déjà dans quelques-uns des flux migratoires.

La deuxième implication se réfère aux regroupements familiaux. Socialement en effet, la transformation de l'immigration des individus en immigration familiale représente un facteur de normalisation des populations immigrées, et donc devrait rassurer la majorité autochtone. Pourtant le regroupement familial est normalement accordé uniquement lorsque l'immigré démontre avoir atteint un niveau suffisant d'intégration économique et peut se loger. Cette condition, concevable comme un facteur permettant de contenir les comportements anomiques et indésirables, est en fait liée à un type de considérations relatives aux possibles dépenses publiques liées à l'arrivée redoutée de familles qui ne sont pas économiquement autosuffisantes ${ }^{15}$. Par conséquent, il se pose un problème de philosophie politique : les immigrants pauvres n'ont-ils pas le droit de vivre avec leur famille ? Ne s'agit-il pas d'un droit humain inaliénable ? Et d'un point de vue des politiques publiques aussi, surgit un dilemme : les familles immigrées sont-elles un coût social à contenir, ou un investissement à promouvoir permettant une immigration plus intégrée ?

15 Considérer l'immigré comme facteur de désordre social se réfère à la population masculine. La femme immigrée seule (et peut-être en situation irrégulière) est au contraire considérée comme moins exigeante, disposée à accepter des conditions de cohabitation avec les pourvoyeurs d'emploi ; elle n'est pas perçue comme socialement dangereuse, excepté éventuellement la composante prise dans la prostitution (Abbatecola, 2006). 
Quant au point le plus sensible de la question, la séparation d'enfants de leurs mères, au lieu d'en délégitimer les souffrances comme l'expression d'une idéologie de la famille et de la maternité inadaptées à l'époque (pour les familles pauvres du Tiers Monde bien sûr, alors que dans les familles riches du Premier Monde le modèle semble plus que jamais à l'apogée), la position de Hondagneu-Sotelo et Avila ne semble plus acceptable : ces auteures demandent que les mères migrantes aient la possibilité de décider de façon autonome des modalités par lesquelles elles souhaitent organiser leurs propres devoirs maternels. Elles ajoutent que cela serait le début de politiques professionnelles et familiales justes, face à l'inégalité non seulement de genre mais aussi de race, de classe et de citoyenneté (1997 : 568).

La troisième implication renvoie aux problèmes de la réorganisation des soins à domicile, dans le sens d'une plus grande attention à la vie et au bien-être des employées de maison, et donc d'horaires et de conditions de travail plus proches de celles des autres travailleurs. Les regroupements sont un facteur important pour imaginer différents régimes d'organisation et pour rechercher des possibilités d'éviter la cohabitation avec les employeurs.

Nous avons vu que le regroupement n'était pas la fin heureuse d'une histoire de séparation et de retrouvaille, de souffrance et d'espoir. Tout au plus c'est un nouveau départ, lui aussi chargé d'inconnu et de défis. Toutefois on ne peut pas en méconnaître la portée plus générale sur la transformation de la société avec les regroupements familiaux et l'augmentation d'une nouvelle génération qui, qu'on le veuille ou non, est destinée à devenir une composante stable et légitime de la société d'accueil. La composition ethnique et culturelle de la population se modifie. La globalisation par le bas, qui a débuté avec l'engagement de femmes étrangères pour assister des personnes âgées, soigner des enfants, entretenir des logements, rendre plus fonctionnelle l'organisation complexe de notre vie quotidienne, est une force sociale destinée à transformer la société dans laquelle nous vivons. 


\section{Références bibliographiques}

ABBATECOLA Emanuela (2006) L'altra donna. Immigrazione e prostituzione in contesti metropolitani, Milano, FrancoAngeli, 160 p.

AMBROSINI Maurizio (2005a) Sociologia delle migrazioni, Bologna, Il Mulino, 294 p.

AMBROSINI Maurizio (2005b) Dentro il welfare invisibile: aiutanti domiciliari immigrate e assistenza agli anziani, Studi emigrazione, XLII, n 159, septembre 2005.

AMBROSINI Maurizio (2008) Un'altra globalizzazione. La sfida delle migrazioni transnazionali, Bologna, Il Mulino, $247 \mathrm{p}$.

AMBROSINI Maurizio et ABBATECOLA Emanuela (A Cura Di) (2004) Immigrazione e metropoli. Un confronto europeo, Milano, Iard-FrancoAngeli.

AMBROSINI Maurizio e COMINELLI Claudia (A Cura Di) (2005) Un'assistenza senza confini. Welfare "leggero", famiglie in affanno, aiutanti domiciliari immigrate, Osservatorio regionale per l'integrazione e la multietnicità, Milano, Regione Lombardia- Fondazione Ismu.

AMBROSINI Maurizio e QUEIROLO PALMAS Luca (A Cura Di) (2005) I latinos alla conquista dell'Europa. Nuove migrazioni e spazi della cittadinanza, Milano, FrancoAngeli.

ANDERSON Bridget (2000) Doing the Dirty Work? The Global Politics of Domestic Labour, London, Zed Books, $224 \mathrm{p}$.

ARANDA Elizabeth M. (2003) Global care work and gendered constraints: the case of Puerto Rican transmigrants, Gender and Society, vol. 17, n 4, pp. 609-626.

BALDASSAR Loretta (2007) Transnational Families and aged care: the mobility of care and the migrancy of ageing, Journal of ethnic and migration studies, vol. 33, n. 2 (March), pp. 275297.

BANFI Ludovica et BOCCAGNI Paolo (2007) Transnational family life: One pattern or many, and why? A comparative study on female migration, Gender, relazione al convegno Generations and the Family in International Migration, European University Institute, Robert Schuman Centre for Advanced Studies.

BASCH Linda, GLICK SCHILLER Nina and SZANTON BLANC Christina (1994) Nations Unbound: Transnational Projects, Postcolonial Predicaments and Deterritorialized NationStates, Amsterdam, Gordon \& Breach, 344 p.

BAUMANN Gerd (1999) The Multicultural Riddle. Rethinking National, Ethnic and Religious Identities, London, Routledge, $192 \mathrm{p}$.

BENÍTEZ J.L. (2006) Transnational dimensions of the digital divide among Salvadoran immigrants in the Washington DC metropolitan area, Global Networks, 2, n 6, pp. 181-199.

BONIZZONI Paola (2007) Famiglie transnazionali e ricongiunte: per un approfondimento nello studio delle famiglie migranti, Mondi migranti, $\mathrm{n}^{\circ}$ 2, pp. 91-108.

CAIRNCROSS Frances (1997) The Death of Distance, London: Orion, 303 p.

CASTAGNONE E., EVE M., PETRILlO E.R. et PIPERNO F. (2007) Madri migranti. Le migrazioni di cura dalla Romania e dall'Ucraina in Italia. Percorsi e impatto sui paesi di origine, CESPI-FIERI, Roma, working paper 34/2007.

CELLA Gian Primo (1997) Le tre forme dello scambio. Reciprocità, politica, mercato a partire da K.Polanyi, Bologna, Il Mulino, 248 p.

CHALOFF J. et PIPERNO F. (2007) L'altra faccia del nostro welfare: il drenaggio di cura nei paesi di origine. Il caso della Romania, Roma, CESPI papers.

COLEN S. (1995) Like a Mother to Them, en Ginsburg F. and Rapp R. (eds), Conceiving the New World Order, Berkeley, University of California Press.

COLOMBO Enzo (2007) L'estranea di casa: la relazione quotidiana tra datori di lavoro e badanti, en Colombo e Semi (a cura di), Multiculturalismo quotidiano. Le pratiche della differenza, Milano, FrancoAngeli, pp. 99-127. 
COLOMBO Enzo e SEMI Giovanni (2007) Multiculturalismo quotidiano. Le pratiche della differenza, Milano, FrancoAngeli, $166 \mathrm{p}$.

LEONARDO Micaela (1987) The Female World of Cards and Holidays: Women, Families and the Work of Kinship, Signs: Journal of women in culture and society, n. 12, pp. 440-453.

DIMINESCU Dana (2005) Le migrant connecté. Pour un manifeste épistémologique, Migrations et société, vol.XVII, n.102, novembre-décembre, pp. 275-292.

DION Karen K. et DION Kenneth L. (2001) Gender and cultural adaptation in immigrant families, Journal of Social Issues, vol. 57, n. 3, pp. 511-521.

DREBY Joanna (2006) Honor and Virtue: Mexican Parenting in the Transnational Context, Gender and Society, vol. 20, n. 1, pp. 32-59.

EHRENREICH Barbara and HOCHSCHILD Arlie Russell (eds) (2002) Global Woman: Nannies, Maids, and Sex Workers in the New Economy, New York, Metropolitan Books, $288 \mathrm{p}$.

EVE M. (2007) Considerazioni introduttive, en Castagnone et al., Madri migranti. Le migrazioni di cura dalla Romania e dall' Ucraina in Italia. Percorsi e impatto sui paesi di origine, pp. 510.

HAGAN John (1999) Social networks, gender and immigrant incorporation: resources and constraints, American Sociological Review, vol.63, n.1 (February), pp. 55-67.

HONDAGNEU-SOTELO Pierrette (1999) Gender and contemporary U.S. immigration, American behavioral scientist, vol. 42, n. 4 (January), pp. 565-576.

HONDAGNEU-SOTELO Pierrette and AVILA Ernestine (1997) I'm here but I'm there: the meanings of Latina transnational motherhood, Gender and Society, vol. 11, pp. 548-71.

LAGOMARSINO F. (2006) Esodi e approdi di genere. Famiglie transnazionali e nuove migrazioni dall'Ecuador, Milano, Fondazione Ismu-FrancoAngeli.

LEVITT Peggy and JAWORSKY Nadya (2007) Transnational migration studies: past developments and future trends, Annual Review of Sociology", vol. 33, pp. 129-156.

MAHLER S.J (2003) Theoretical and empirical contributions. Toward a research agenda for transnationalism in Guarnizo and Smith (eds) Transnationalism from below, vol. 6 Comparative Urban and Community Research, pp. 64-100.

MAHLER S.J (2001) Transnational relationships: the struggle to communicate across borders, Identities, vol. 7, n. 4, pp. 583-619.

PARREÑAS Rhacel Salazar (2001) Servants of globalization. Women, migration, and domestic work, Stanford (Calif), Stanford University Press, 328 p.

PARREÑAS Rhacel Salazar (2005) Children of global migration. Transnational families and gendered woes, Stanford (Calif.), Stanford University Press, 224 p.

PESSAR Patricia and MAHLER Sarah (2003) Transnational migration: bringing gender, International Migration Review, vol. 37, n. 3 (Fall), pp. 812-846.

PORTES Alejandro (1999) Conclusion: towards a new world - the origins and effects of transnational activities, Ethnic and racial studies, vol. 22, n. 2, pp. 463-477.

PORTES Alejandro (2003) Conclusion: theoretical convergencies and empirical evidence in the study of immigrant transnationalism, International Migration Review, vol. 37, n. 3 (Fall), pp. 874-892.

PRIBILSKY J. (2003) Aprendemos a convivir: conjugal relations, co-parenting and family life among Ecuadorian transnational migrants in New York city and the Ecuadorean Andes, Global Networks, vol. 4, n 3, pp. 313-334.

SEMI Giovanni (2007) Teorie multiculturali: approcci normativi, studi idiografici e l'ingombrante presenza del quotidiano, en Colombo e Semi (2007), Multiculturalismo quotidiano. Le pratiche della differenza, pp. 37-56.

SIGNORELLI Amalia (2006) Migrazioni e incontri etnografici, Palermo, Sellerio, 243 p. 
SUAREZ-OROZCO C., TODOROVA I.L.G. e LOUIE J. (2002) Making up for lost time: the experience of separation and reunification among immigrant families, Family Process, vol. 41, n. 4, pp. 625-643.

THOMPSON Paul et BAUER Elaine (2005) Sources of aid and resilience and points of pain in Jamaican migrant families, Revue européenne des migrations internationales, a.21, n. 3, pp. 9-26.

TOGNETTI BORDOGNA Mara (2005) Ricongiungere la famiglia altrove, Milano, FrancoAngeli.

TORREALBA ORELLANA R. (1989) Migratory movements and their effects on family strucature: the latin american case, International Migrations, n. 2 (June).

VERTOVEC Steven (2004a) Migrant transnationalism and modes of transformation, in International migration review, vol. 38, n. 3 (Fall), pp. 970-1001.

VERTOVEC Steven (2004b) Cheap calls: the social glue of migrant transnationalism, Global Networks, a.4, n. 2, pp. 219-224.

WILDING Raelene (2006) 'Virtual' intimacies? Families communicating across Transnational Contexts, Global Networks, a.2, n. 6, pp. 125-142.

ZANFRINI Laura e ASIS Marja M.B. (A Cura Di) (2006) Orgoglio e pregiudizio. Una ricerca tra Filippine e Italia sulla transizione all' età attiva dei figli di emigrati e dei figli di immigrati, Milano, Fondazione Ismu-FrancoAngeli. 


\title{
Séparées et réunies : familles migrantes et liens transnationaux
}

\author{
Maurizio AMBROSINI
}

\begin{abstract}
Cet article s'intéresse à la demande de main-d'œuvre féminine, en Italie et plus généralement en Europe méridionale, qui produit ce que l'on peut définir comme la «stratification internationale des opportunités de soins et d'assistance ». Il aborde la façon dont les mères séparées de leurs enfants à cause de l'émigration s'efforcent de continuer à exercer leurs devoirs maternels, malgré les frontières et les distances, mettant en évidence les différences repérées sous la forme d'une typologie des familles transnationales.

L'auteur montre que le phénomène n'est pas rigide et bloqué. Il présente un aspect évolutif, qui transforme des familles transnationales en familles réunies, dans lesquelles par ailleurs surgissent de nouveaux problèmes. Dans sa conclusion, l'article discute des implications théoriques et politico-sociales des opportunités d'assistance et du care drain.
\end{abstract}

\section{Separated and Reunited: Migrant Families and Transnational Bonds}

\section{Maurizio AMBROSINI}

The article treats the demand of women workforce in Italy and in Southern Europe, hired to satisfy the need of care activities in the families, and producing an international stratification of family care. Then it talks about the ways in which mothers separated from their children try to continue to perform their parental duties, notwithstanding frontiers and distances, and shows the differences that exist among transnational families in the form of a typology.

The analysis continues observing that the phenomenon of transnational families is not rigid and fix. It contains an evolving component, transforming a part of transnational families in reunited families, in which new problems arise. In the conclusion, the author discusses the theoretical and socio-political implications of the question.

\section{Separadas y reunidas: familias migrantes y lazos transnacionales}

\section{Maurizio AMBROSINI}

Este artículo trata de la demanda de maniobra femenina en Italia y más generalmente en Europa del sur, defendiéndola como la "estratificación internacional de las oportunidades de cuidado y de asistencia". Aborda la manera en que las madres, separadas de sus hijos por la emigración, tratan de seguir cumpliendo con sus deberes maternales a pesar de las fronteras y de las distancias. Una tipología de las familias transnacionales pone en valor las diferencias entre las prácticas de estas madres.

El autor continua mostrando que el fenómeno no es ni definitivo, ni bloqueado. Presenta las evoluciones que transforman una parte de las familias transnacionales en familias reunidas, en las cuales surgen nuevos problemas. Concluye su artículo discutiendo de las implicaciones teóricas y politico-sociales de la asistencia y del care drain. 